\title{
Chiral Alkyl Amine Synthesis via Catalytic Enantioselective Hydroalkylation of Enamides
}

Deyun Qian, Srikrishna Bera, and Xile Hu*

Laboratory of Inorganic Synthesis and Catalysis, Institute of Chemical Sciences and Engineering, École Polytechnique Fédérale de Lausanne (EPFL), ISIC-LSCI, BCH 3305, Lausanne 1015, Switzerland

E-mail: xile.hu@epfl.ch

\begin{abstract}
Chiral alkyl amines are omnipresent as bio-active molecules and synthetic intermediates. Catalytic and enantioselective synthesis of alkyl amines from readily accessible precursors is challenging. Here we develop a nickel-catalyzed hydroalkylation method to assemble a wide range of chiral alkyl amines from enamides and alkyl halides in high regioand enantioselectivity. The method works for both non-activated and activated alkyl halides, and is able to produce enantiomerically enriched amines with two minimally differentiated $\alpha$ alkyl substituents. The mild conditions lead to high functional group tolerance, which is demonstrated in the post-product functionalization of many natural products and drug molecules, as well as the synthesis of chiral building blocks and key intermediates to bio-active compounds.
\end{abstract}




\section{Introduction}

Enantiomerically pure amines are frequently encountered in natural products, pharmaceuticals, and agrochemicals (Fig. 1A). ${ }^{1}$ They are also important building blocks and chiral auxiliaries in asymmetric synthesis. General, catalytic, and enantioselective assembly of chiral amines, especially those with two minimally differentiated aliphatic substituents, represents a synthetic challenge. ${ }^{1 \mathrm{c}}$ Although chiral amines can be prepared by hydrogenation of imines, enamines, and their derivatives using precious metal catalysis (Fig. 1B), the catalysts are costly and the substrates typically have an $\alpha$-aryl or $\alpha$-carboxyl substituent. ${ }^{2}$ Likewise, enantioselective addition of an alkyl organometallic reagent ${ }^{1 b, 3}$ or an alkyl radical ${ }^{1 b, 4}$ to imine derivatives is mostly applicable for the synthesis of chiral amines with one $\alpha$-aryl or $\alpha$-carboxyl group, in addition to often require either highly activated substrates or high catalyst loadings (Fig. 1C and 1D). Recent breakthroughs in $\mathrm{CuH}$-catalyzed asymmetric hydroamination of internal alkenes provide a new approach to chiral amines synthesis (Fig. 1E). ${ }^{5}$ Nevertheless, trisubstituted, tetrasubstituted, and cis-disubstituted alkenes and alkenes bearing electrondonating substituents are still difficult substrates, limiting the types of chiral amines that can be prepared from this approach. ${ }^{5,6}$

$\mathrm{Ni}$-catalyzed hydroalkylation of alkenes ${ }^{7}$ has emerged as an attractive alternative to traditional alkyl-alkyl cross-coupling. ${ }^{8}$ By using stable and abundant alkenes as pro-nucleophiles instead of reactive organometallic reagents, hydroalkylation offers substantial advantages in practicality, scope, and functional group compatibility over conventional cross-coupling. Several elegant examples of asymmetric hydroalkylation to create a new stereocenter at the carbon originated from activated alkyl electrophiles have been reported. ${ }^{9}$ However, enantioselective hydroalkylation to introduce a new stereocenter at the carbon originated from the alkenes is challenging due to the tendency of $\mathrm{Ni}-\mathrm{H}$ to mediate chain walking of an internal alkene to form a terminal, non-chiral Ni-alkyl species. ${ }^{10}$ By installing an $\alpha$-directing group such as boryl and aryl, our group ${ }^{11}$ and the group of $\mathrm{Zhu}^{12}$ were recently able to develop $\mathrm{Ni}$ catalyzed enantioselective hydroalkylation and hydroarylation for the synthesis of chiral alkyl boronates and 1,1-diarylalkanes, respectively. The electron-withdrawing $\alpha$-directing groups are essential in stabilizing the branched alkyl-Ni intermediates against chain walking. In this context, we envisioned enantioselective hydroalkylation of enamine derivatives for the synthesis of chiral amines (Fig. 1F). If successful, this approach would be general and efficient in introducing a diverse set of alkyl groups at the $\alpha$-position of chiral amines, a task difficult to achieve using existing methods. Moreover, because enamines can be easily prepared from readily available alkyl aldehydes or other precursors, ${ }^{13}$ the approach will have a wide scope or be more practical than hydrogenation of imines which require less accessible dialkyl ketones as reagents. However, an $N$-based $\alpha$-substituent as in enamine derivatives is typically considered as an electron-donating group. ${ }^{2 \mathrm{~b}}$ When we started there was no prior report that such a group would stabilize a branched alkyl-Ni intermediate to direct enantioselective hydroalkylation, although a report of Ni-catalyzed enantioselective hydroarylation of $N$-vinyl amides appeared while we were writing this manuscript. ${ }^{14}$

Here we describe the development of a modular method based on this approach. The method allows the enantioselective coupling of a wide range of $\mathrm{Cbz}$-enamides $(\mathrm{Cbz}=$ benzyloxycarbonyl) with both activated and non-activated alkyl halides to give enantiomerically enriched $\alpha$-alkyl chiral amines. The method has high group tolerance, and can be used for the functionalization of many natural products and bio-active compounds, as well as the synthesis of valuable chiral intermediates. 
A) Representative alkaloids and drugs demonstrating the ubiquitous nature of chiral alkyl amines

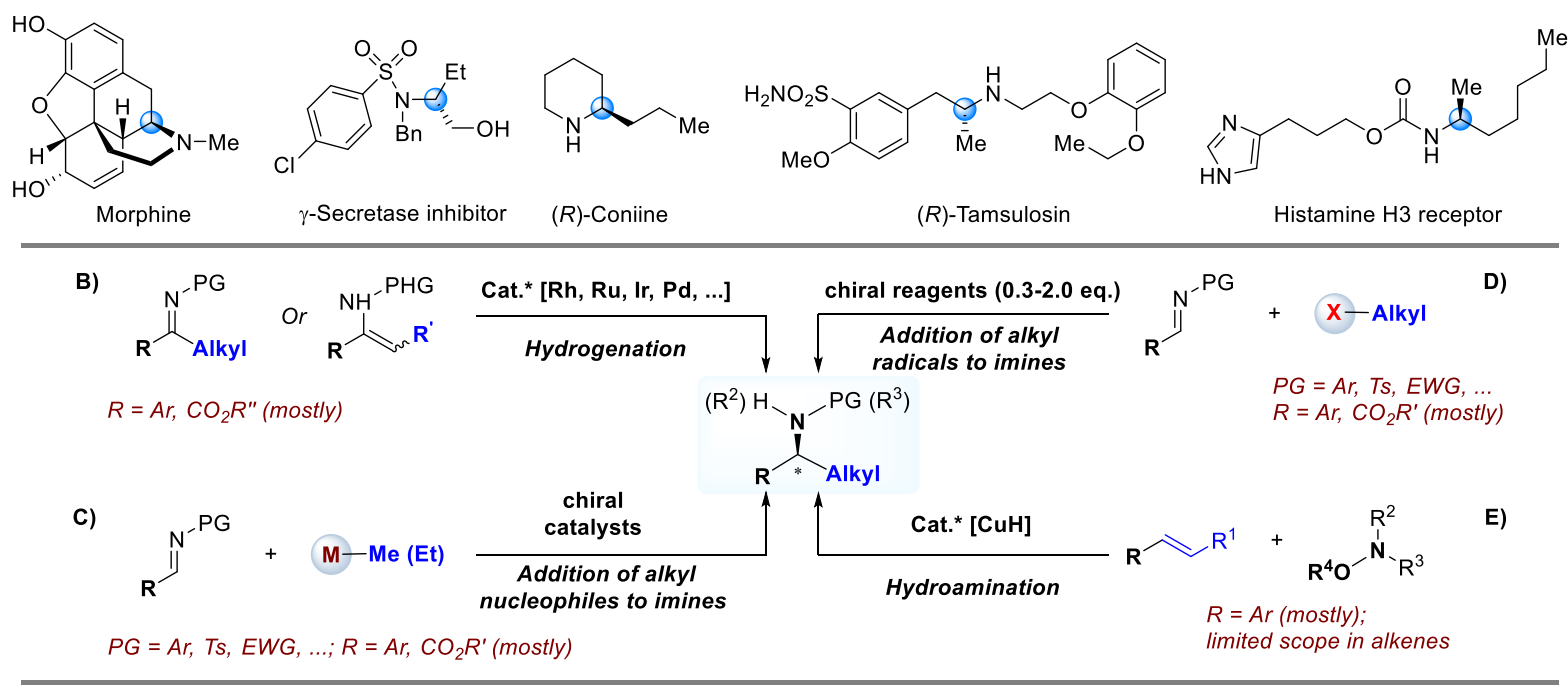

F) Hydroalkylation of enamines

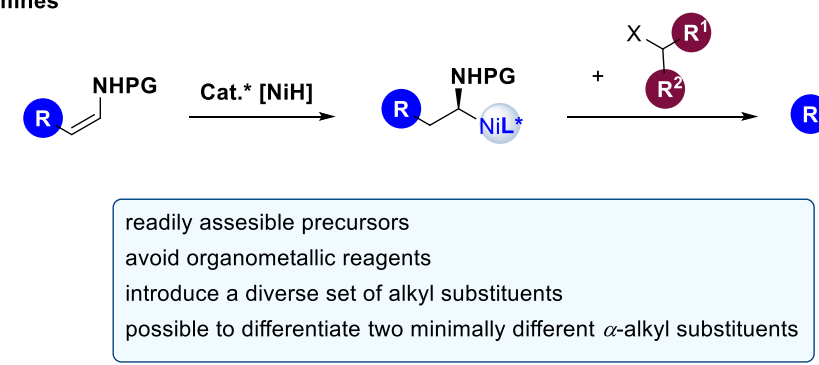

Figure 1. Significance of chiral $\alpha$-alkyl amines and representative methods of their synthesis.

\section{Results.}

We started the development by optimizing hydroalkylation of benzyl (Z)-prop-1-en-1ylcarbamate (1a, 1.0 equiv.) using iodocyclohexane (2a, 1.5 equiv.) as the alkylating agent, a silane or hydroborane (2.0 equiv.) as the hydride source, a nickel salt (10 mol\%) in combination with a chiral nitrogen-based ligand $(15 \mathrm{~mol} \%)$ as the catalyst (Table 1). Initial screening suggested room temperature and 40 hours as appropriate conditions. The influence of ligands, Ni salts, hydride sources, bases, solvents, as well as the outcome of control experiments are described in Supplementary Tables 1-6. A key summary is shown in Table 1. With diethoxymethylsilane (DEMS) as the hydride source, 2,2-bis(2-oxazoline) (Bi-Ox) and pyridine-oxazoline (Py-Ox) ligands were effective, giving the desired product 3a in various yields and enantiomeric ratio (er), whereas bis-oxazoline (Box), pyridine bis-oxazoline (Pybox), and phosphinooxazoline (Phox) ligands gave no product (Supplementary Table 1). A large number of Bi-Ox ligands were screened, revealing the sensitivity of reaction outcome on the substituents of the ligands. Aryl substituents typically gave higher ers than alkyl substituents (Entries 1-5, Table 1), and the best result was obtained with the phenyl-substituted Bi-Ox ligand $\mathrm{L}^{*} 1$, which gave a yield of 91\% and an er of 90:10 (Entry 1, Table 1). $\mathrm{NiBr}_{2}$.diglyme was the best Ni source among various Ni(II) salts (see Supplementary Table 2). Several hydrosiloxanes could be used as the hydride source with similar efficiency, but less electrophilic hydrosilanes such as $\mathrm{Et}_{3} \mathrm{SiH}$ and $\mathrm{Ph}_{2} \mathrm{MeSiH}$ were inefficient (Supplementary Table 3). The enantiomeric ratio was increased to 92:8 using pinacolborane (HBpin) as the hydride source (Entry 6, Table 1) while the yield remained as high as $85 \%$. A slighly lower er 
but much lower yield was otbained using catecholborane (HBcat) (Entry 7, Table 1). Different bases gave similar enantioselectivities but very different yields, and KF was the best base (Supplementary Table 4). The yields, and to a less degree, the ers, were sensitive to the solvents (Supplementary Table 5). A meticulous screening revealed $N, N{ }^{\prime}$ dimethylpropyleneurea (DMPU) as the best solvent (entry 8, Table 1). Thus, the final optimized conditions were identified as $\mathrm{NiBr}_{2} \bullet$ diglyme (10 mol\%) plus $\mathrm{Bi}-\mathrm{Ox} \mathbf{L}^{*} \mathbf{1}(15 \mathrm{~mol} \%)$ as the catalyst, HBpin (2.0 equiv.) as the hydride source, KF (2.0 equiv.) as the base, and DMPU as the solvent. Under these conditions, 3a was obtained as a single regioisomer in $92 \% \mathrm{GC}(87 \%$ isolated) yield with an er of 94:6 (entry 8, Table 1). Compared to Ni-catalyzed hydroalkylation of alkenyl boronates, ${ }^{11}$ the best chiral ligand is the same while the optimized Ni salt, hydride source, and solvent are all different, indicating a strong influence of the directing group in the reactivity. Two recent reports describe Ni-catalyzed enantioselective hydroarylation of alkenes where the best ligands were based on bisimidazolines, ${ }^{12,14}$ a different class of ligands compared to the Bi-Ox used here. This difference suggests a mechanistic divergence in these related reactions.

\section{Table 1. Summary of the effects of key reaction parameters ${ }^{\mathrm{a}}$}

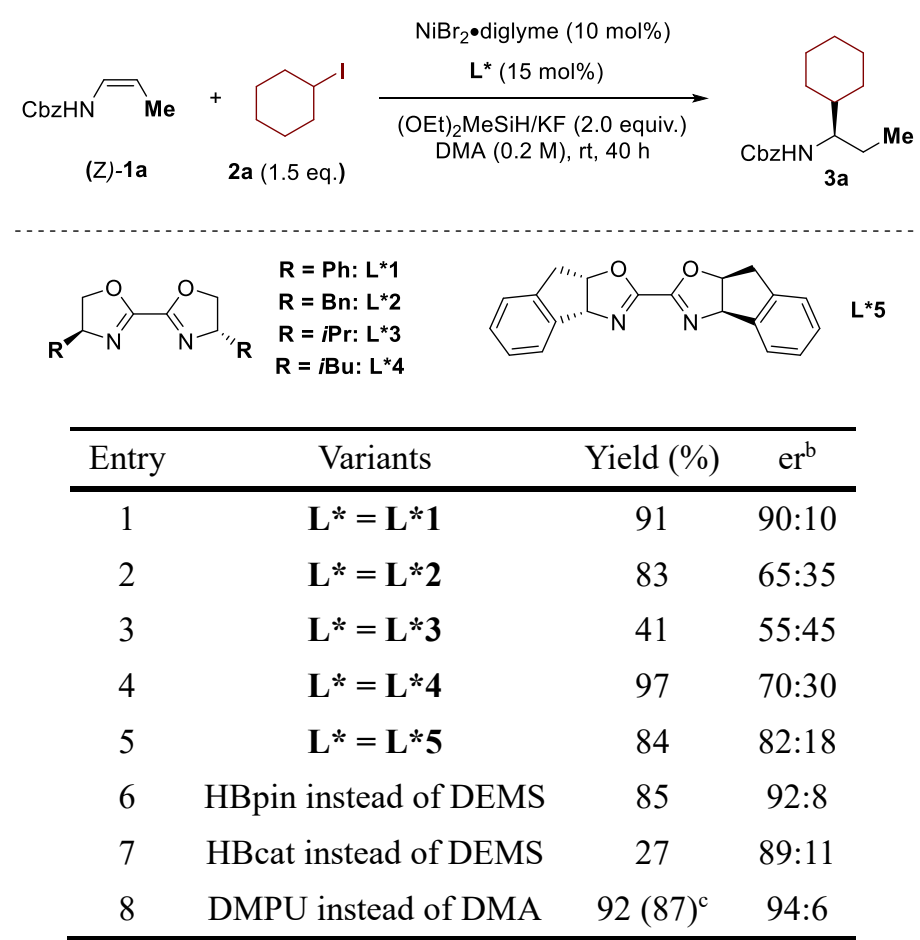

${ }^{\text {a }}$ See the Supplementary Information (section 2) for experimental details; all reactions were carried out in a 0.1 mmol scale with respect to 1a; corrected GC yields using $n$-dodecane as an internal standard were reported. ${ }^{\mathrm{b}}$ The er values were determined by HPLC analysis. ${ }^{\mathrm{c}}$ Isolated yield. DMA $=$ Dimethylacetamide; DEMS = diethoxymethylsilane; DMPU $=N, N^{\prime}$-dimethylpropyleneurea; $\mathrm{Cbz}=$ benzyloxycarbonyl; $\mathrm{rt}=$ room temperature. 
Table 2. Scope of Ni-catalysed enantioselective hydroalkylation of enamides ${ }^{\text {a }}$
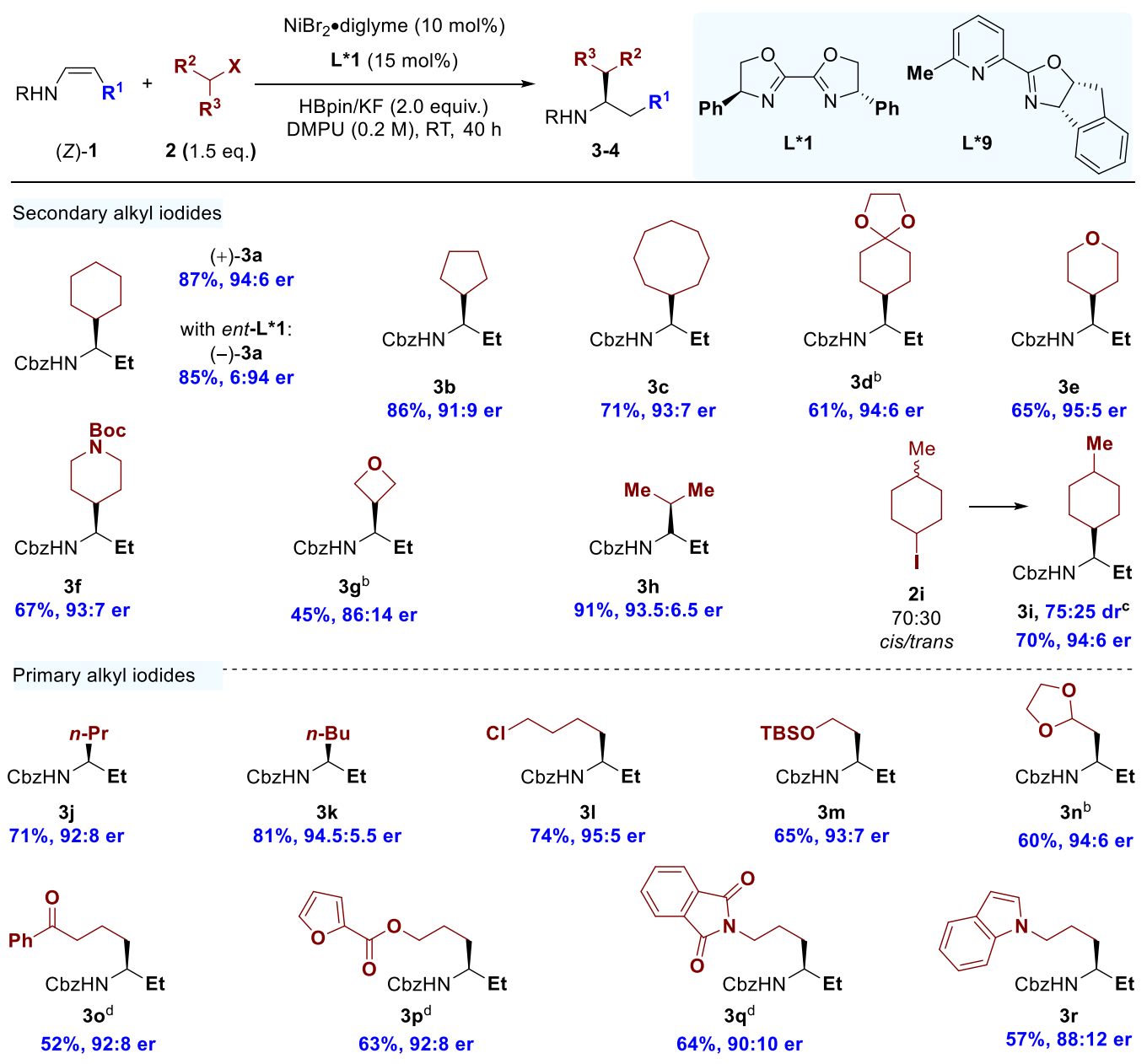

Benzylic bromides
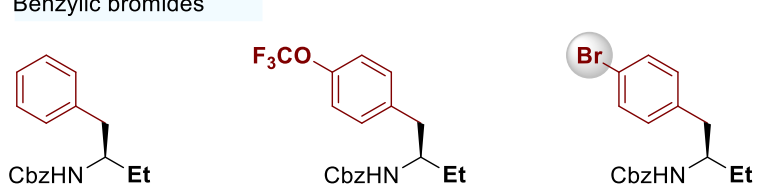

3 s
$92 \%, 95: 5$ er

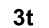

$82 \%, 95: 5$ er

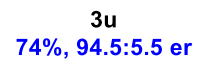

$64 \%, 90: 10$ er

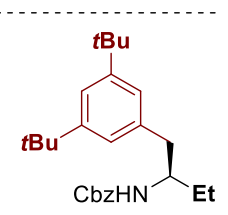

Enamides<smiles>CCCCC(NC)C1CCCCC1</smiles>

$4 a$
$63 \%, 94: 6$ er<smiles>COC(=O)NC(CCCCC(C)(C)C)C(C)(C)C</smiles>

$4 \mathrm{~g}$
$72 \%, 94: 6$ er

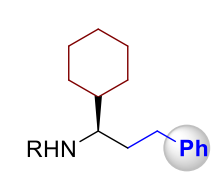

4b, R = Cbz: $83 \%, 95: 5$ er $4 c, R=$ Boc: $82 \%, 86: 14$ er

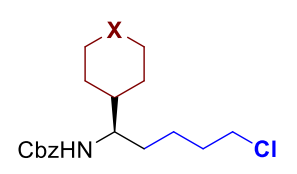

$4 \mathrm{~d}, \mathrm{X}=\mathrm{CH}_{2}: 65 \%, 95: 5 \mathrm{er}$ $4 \mathrm{e}, X=$ NBoc: $72 \%, 93: 7 \mathrm{er}$

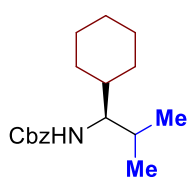

$4 \mathbf{i}$

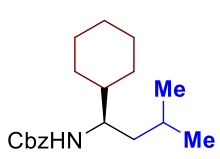

$72 \%, \stackrel{4 h}{93: 7}$ er<smiles>CCC(Cc1cccc(C#N)c1)NC(=O)OCc1ccccc1</smiles>

$3 \mathbf{v}$

$3 v$
$95: 5$ er
$3 w$

95\%, 94:6 er

Conditions: a All reactions were carried out with $\mathrm{NiBr}_{2} \bullet$ diglyme (15 mol\%), ligand $\mathbf{L} * 1$ (15 mol\%), 1 (0.20 mmol), $2(0.30 \mathrm{mmol})$, HBpin $(0.40 \mathrm{mmol}), \mathrm{KF}(0.40 \mathrm{mmol})$ and DMPU $(1.0 \mathrm{~mL})$ at room temperature for 40 hours. ${ }^{\mathrm{b}}$ $(\mathrm{OEt})_{2} \mathrm{MeSiH}$ instead of HBpin. ${ }^{\mathrm{c}}$ The dr value was determined by ${ }^{1} \mathrm{H}$ NMR and HPLC analysis. ${ }^{\mathrm{d}}$ DMA instead of DMPU. ${ }^{\mathrm{e}} \mathrm{NiI}_{2} \bullet \mathrm{XH}_{2} \mathrm{O}, \mathbf{L} * \mathbf{9}$ and DMA instead of the corresponding standard parameters. ${ }^{\mathrm{f}} \mathbf{1 i} / \mathbf{2} \mathbf{a}=1.5: 1$; $\mathrm{NiI}_{2} \bullet \mathrm{xH}_{2} \mathrm{O}, \mathbf{L} * \mathbf{9},(\mathrm{OEt})_{3} \mathrm{SiH}$ and DMA instead of the corresponding standard parameters. 
We then explored the scope of this enantioselective hydroalkylation method (Table 2). The method worked well for the coupling of various secondary alkyl iodides, including both acyclic and cyclic substrates, to give the corresponding chiral dialkyl amines with good yields and high enantioselectivity (3a-3i). These products would be difficult to access via enantioselective cross-coupling, ${ }^{8 b, 8 \mathrm{c}}$ which is difficult for the coupling of two secondary alkyl fragments. ${ }^{15}$ Cyclic groups relevant to medicinal chemistry such as $2 \mathrm{H}$-pyran, piperidine and oxetane were well tolerated (3e-3g). Hydroalkylation with $2 \mathbf{i}$ had a good yield and er, but with moderate diastereoselectivity (3i).

The hydroalkylation method worked for primary alkyl iodides as well. Functional groups such as chloride (3l), ether (3m), acetal (3n), ketone (3o), ester (3p), furan (3p), phthalimide (3q), indole (3r), and amine (3r) were all compatible. Besides non-activated alkyl halides, activated halides such as benzylic bromides were also suitable substrates for the hydroalkylation, yielding the corresponding chiral amines in good yields and high enantioselectivity (3s-3w). The absolute configuration of $\mathbf{3 s}$ was determined as $(R)$ by comparing its optical rotation value to a reported compound (Supplementary Section 9). We assigned the same absolute configuration to all analogous products. The reactions were insensitive to the electronic properties of the substituents of the benzylic bromides. An aryl bromide group was tolerated in the reaction, $(\mathbf{3 u})$, reserving a reaction site for further down-stream cross-coupling. The coupling of unactivated tertiary alkyl iodides such as tert-butyl iodide was unsuccessful.

An array of enamides could be used as the pro-nucleophiles for the enantioselective hydroalkylation $(\mathbf{4 a}-\mathbf{4 j})$. Not only $\mathrm{CbzNH}$, but also BocNH $(\mathbf{4 c}$ and $\mathbf{4 e} ; \mathrm{Boc}=t$ butoxycarbonyl), was a viable directing group. The $\mathrm{C}-\mathrm{C}$ bond formation was regioselective at the carbon $\alpha$-to the RNH group, even in the presence of an aryl group (4b and $\mathbf{4 c}$ ). Functional groups such as alkyl chloride (4d and $\mathbf{4 e})$ and ester $(\mathbf{4 f}$ and $\mathbf{4 g})$ on enamides were tolerated. Coupling of a sterically demanding $\beta, \beta^{\prime}$-disubstituted enamide had a modest yield ( $48 \%$ ) but a high er (94:6) (4i). Vinylamine, a synthetically useful substrate that poses a challenge in regioselectivity, ${ }^{16}$ was coupled to give a single regioisomer $(\mathbf{4 j})$ with a high yield and high enantioselectivity with modified reaction conditions.

\section{Table 3. Reactions of substrates derived from natural products and drugs ${ }^{\mathrm{a}}$}

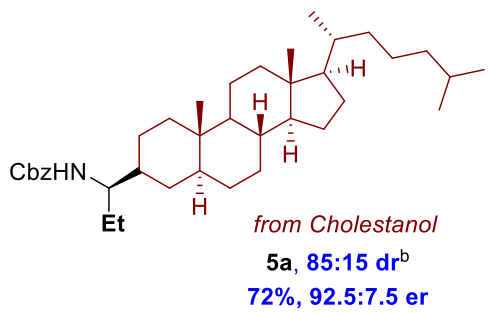

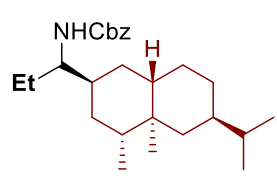

from Nootkatone 5b, 80:20 dr ${ }^{b}$ $81 \%, 94: 6$ er

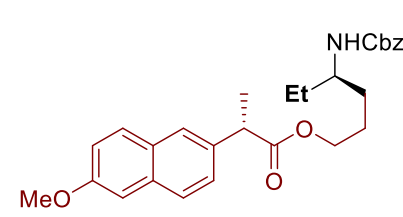

from Naproxen 5 c $76 \%, 91: 9 \mathrm{dr}$

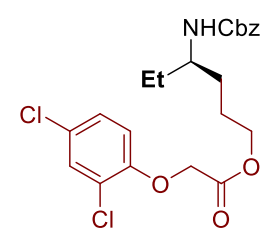

from 2,4-D

$5 d$ $56 \%, 92: 8$ er
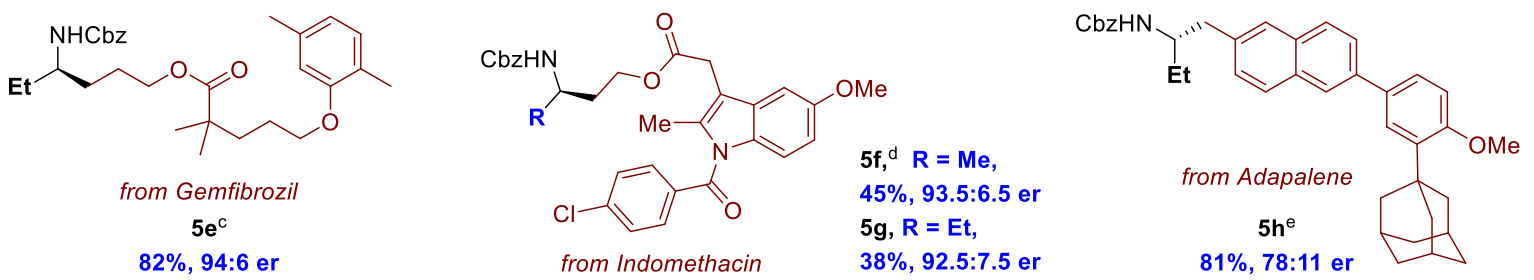

Conditions: a All reactions were carried out with $\mathrm{NiBr}_{2} \bullet$ diglyme (15 mol\%), ligand $\mathbf{L} * 1$ (15 mol\%), 1 (0.20 mmol), $2(0.30 \mathrm{mmol})$, HBpin $(0.40 \mathrm{mmol}), \mathrm{KF}(0.40 \mathrm{mmol})$ and DMPU $(1.0 \mathrm{~mL})$ at room temperature for 40 hours. ${ }^{b}$ The dr value was determined by ${ }^{1} \mathrm{H}$ NMR and HPLC analysis. ${ }^{c}$ DMA instead of DMPU. ${ }^{d} \mathrm{NiI}_{2} \bullet \mathrm{xH}_{2} \mathrm{O}, \mathbf{L}^{* 9}$, $(\mathrm{OEt})_{3} \mathrm{SiH}$, and DMA instead of the corresponding standard parameters. ${ }^{\mathrm{e}}$ ent- $\mathbf{L} * \mathbf{1}$ instead of $\mathbf{L} * \mathbf{1}$. 
The enantioselective hydroalkylation method worked for alkyl halide substrates derived from natural products and drugs, generating chiral alkyl amines bearing the corresponding complex or bio-active alkyl fragments (Table 3). ${ }^{17}$ Alkyl iodides derived from cholestanol, a biomarker (5a) ${ }^{17 a}$ nootkatone, a sesquiterpenoid (5b),${ }^{17 b}$ and naproxen, a nonsteroidal anti-inflammatory drug $(\mathbf{5 c}),{ }^{17 \mathrm{c}}$ which contained one or more stereocenters, reacted to give the corresponding chiral alkyl amines in good yields, high enantioselectivity, as well as modest to good diastereoselectivity. Alkyl iodides derived from drugs such as gemfibrozil (5e), indomethacin (5f and 5g), and adapalene (5h) as well as from a herbicide 2,4-D (5d) were viable reaction partners.

The chiral alkyl amines produced from the above hydroalkylation method proved to be useful synthetic intermediates. ${ }^{18}$ For example, simple silyl ether-deprotection of $\mathbf{3 m}$ afforded the amino alcohol $\mathbf{6}$ in high yield. Compound $\mathbf{6}$ is a common synthon in asymmetric organic synthesis because its $\mathrm{OH}$ moiety can be easily converted to other functional groups without erosion in enantiomeric excess, for instance, in the synthesis of a chiral piperidine (7a) ${ }^{18 a}$ and a $\beta$-amino acid (7b). ${ }^{18 \mathrm{~b}}$ In another example, Finkelstein reaction of $\mathbf{3 l}$ followed by base treatment provided a $(R)$-coniine analogue $(8)$ in 53\% yield and 94:6 er. Chiral amine (-)-3s was a reported intermediate to compound $(R)$-1,2,3,4-tetrahydroisoquinoline $(\mathbf{1 0})$, an inhibitor of phenylethanolamine $N$-methyltransferase. Previously (-)-3s was prepared in three steps from 9 which was in turn prepared by organocatalytic conjugate addition of a nitroalkane to vinylsulfone. ${ }^{18 \mathrm{c}}$ With the current method, (-)-3s was prepared in one step from enamide $\mathbf{1 a}$ and benzyl bromide.

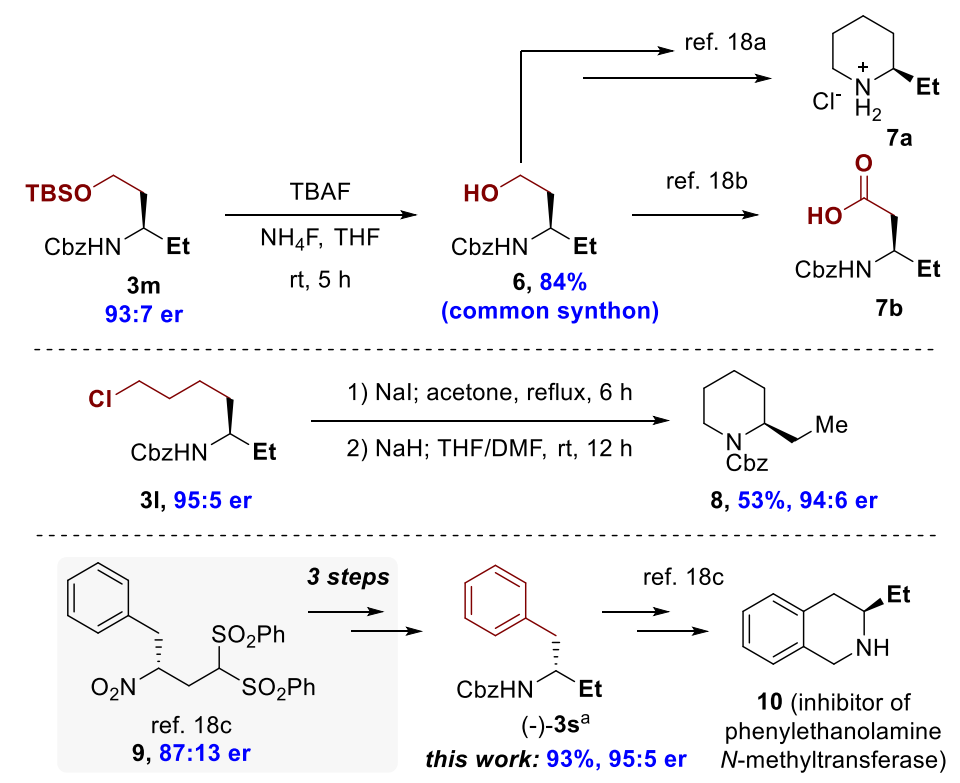

Figure 2. Synthetic applications. See Supplementary Information section 7 for full details. TBAF = Tetrabutylammoniumfluoride. ${ }^{\text {a }}$ With ent $-\mathbf{L} * \mathbf{1}$.

Several experiments were conducted to probe the origin of the enantioselectivity in the present hydroalkylation reactions. When the $(E)$-isomer of $\mathbf{1 a}$ was subjected to the standard conditions (Entry 8, Table 1), 3a was produced just as using (Z)-1a, but both the yield and er were lower than using (Z)-1a (Figure 3a). This result is consistent with Ni-H insertion into alkene as the enantio-determining step. If reductive elimination is the enantio-determining step, a similar er is expected for reactions using either $(Z)$-1a or $(E)-\mathbf{1 a}$. When the allylic amine derivative $\left(\mathbf{1 a}^{\prime}\right)$ was used as the pro-nucleophile, 3a was also produced, albeit in a lower yield and er, together with other regioisomers (Figure $3 b$ ). This result indicates chain walking of the distal alkenyl group mediated by $\mathrm{Ni}-\mathrm{H}$ to form an $\alpha$-CbzHN-stabilized Ni-alkyl species. When an $\mathrm{N}$-methyl 
enamide (Z)-1a" was used as the substrate, almost no hydroalkylation occurred (Figure 3c), indicating an essential role of the $\mathrm{NH}$ group in enamide. To probe the possibility of Nicatalyzed hydroboration of enamide followed by alkyl-alkyl Suzuki cross-coupling, we treated the relevant hydroboration product to the hydroalkylation reaction. However, no crosscoupling occurred, ruling out the Suzuki coupling pathway (Figure 3d). When 1.0 equiv. of (2,2,6,6-tetramethylpiperidin-1-yl)oxyl (TEMPO), a radical scavenger was added to the reaction mixture, the conversion was decreased to $54 \%$ and the yield was decreased to $12 \%$ while the er remained the same (Figure 3e). This result is consistent with the intermediate of an alkyl radical in the hydroalkylation reaction, which was inhibited by TEMPO. Meanwhile, hydrogenation product was obtained in $31 \%$ yield, suggesting that the Ni-alkyl species formed upon $\mathrm{Ni}-\mathrm{H}$ insertion underwent mostly $\mathrm{C}-\mathrm{H}$ reductive elimination instead of alkyl-alkyl coupling in the presence of TEMPO. The reaction progress of $(Z)-\mathbf{1 a}$ with $\mathbf{2 a}$ was monitored over time (Figure 3f). While the yield increased gradually over the course of the reaction, the er remained at about 94:6. This result ruled out a kinetic resolution mechanism. Moreover, the enantiomeric excess of the product and catalyst followed a linear relationship, indicating a monomeric nature of the active catalyst (Figure $3 \mathrm{~g}$ ).
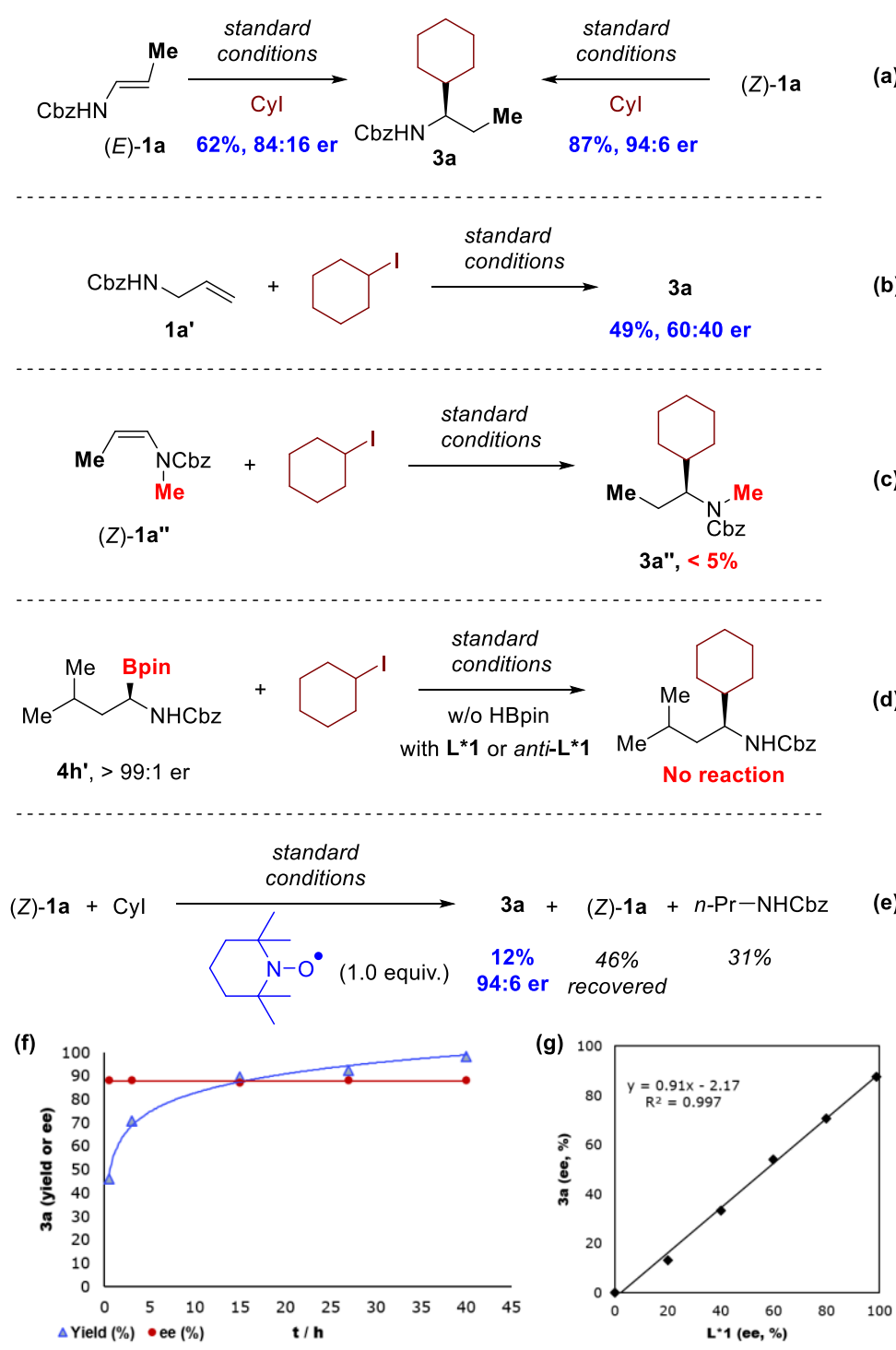

Figure 3. Results of mechanistic experiments. 


\section{Discussion}

Following previous proposals on Ni-catalyzed hydroalkylation ${ }^{9,11}$ and considering the results above, we propose a tentative catalytic cycle for the present enantioselective hydroalkylation of enamides (Figure 4). Under the reaction conditions, a chiral $\mathbf{L}^{*} \mathrm{Ni}^{(\mathrm{I})}-\mathrm{X}$ species $(\mathbf{A})$ is formed as the actual catalyst, which undergoes a single electron transfer (SET) with an alkyl iodide to generate an alkyl radical and $\mathrm{XL} * \mathrm{Ni}^{(\mathrm{II})}-\mathrm{I}(\mathbf{B})$. The latter reacts with a hydride source (e.g, HBpin $+\mathrm{KF}$ ) to generate a $\mathrm{Ni}-\mathrm{H}$ species $\mathrm{XL} * \mathrm{Ni}^{(\mathrm{II})}-\mathrm{H}(\mathbf{C})$ that inserts to the enamide. The resulting Ni-alkyl intermediate (D) then recombines with the alkyl radical to give a formal $\mathrm{Ni}$ (III) complex (E), which undergoes reductive elimination to give the product and regenerate the $\mathrm{Ni}(\mathrm{I})$ catalyst. The carbonyl group of the NHCbz group likely plays a key role in the regioand enantioselectivity of the insertion by coordinating to the Ni center. ${ }^{16 \mathrm{~b}}$ The addition of hydride into the carbon $\beta$ - to the NHCbz group is favoured over the insertion into the $\alpha$-carbon due to the formation of a more stable 5-membered nickelacycle compared to a 6-membered nickelacycle in the latter case. The enantioselectivity of insertion is dictated by the steric interaction of the phenyl substituent with the coordinated enamide. In the favoured transition state, the phenyl group is on the same side as the $\alpha$-hydrogen atom (TS-1a). In the opposite configuration, the phenyl group would experience steric repulsion from the NHCbz group (TS1b). The details of the catalytic cycle are subjected to a dedicated study.

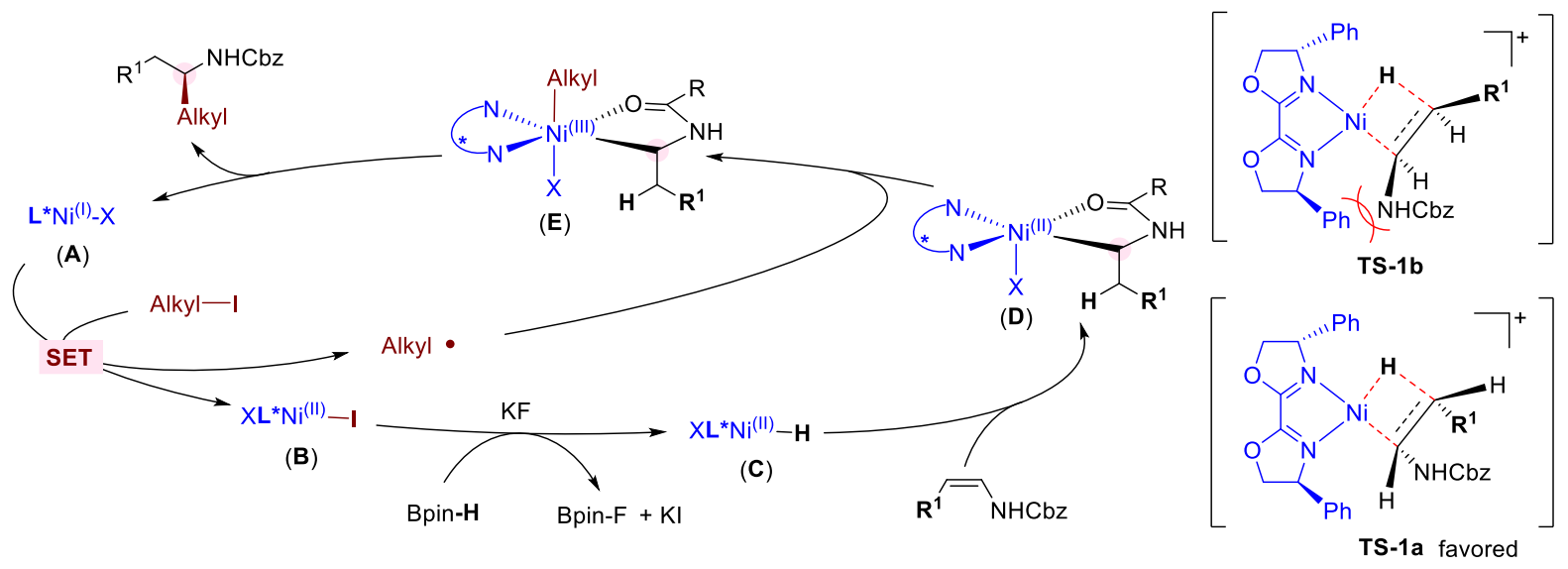

Figure 4. A proposed catalytic cycle.

\section{Conclusion}

In summary, we have developed a method for Ni-catalyzed enantioselective hydroalkylation of enamides. This method allows the synthesis of a wide range of enantiomerically enriched chiral alkyl amines from readily available and stable alkenes while avoiding sensitive organometallic reagents. The method operates under mild conditions and has high functional group tolerance. It has been applied for the post-functionalization of many natural products and drug molecules.

\section{Data Availability}

All data supporting the findings of this study, including experimental procedures and compound characterization, NMR and HPLC plots are available within the Article and its Supplementary Information.

\section{Acknowledgements}


This work is supported by the Swiss National Science Foundation.

\section{Author Contributions}

D.Q. and X.H. conceived the project. D.Q. designed and optimized the synthetic method, and studied the scope, application as well as mechanism. S.B. participated in the study of the scope. All authors analyzed the data. D.Q. and X.H. wrote the manuscript. X.H. directed the research.

\section{Competing Interests}

The authors declare no competing interests.

\section{References}

(1) (a) Nugent, T. C.: Chiral amine synthesis: methods, developments and applications; John Wiley \& Sons, 2010; (b) Li, W.; Zhang, X.: Stereoselective formation of amines; Springer, 2014; (c) Trowbridge, A.; Walton, S. M.; Gaunt, M. J. New Strategies for the Transition-Metal Catalyzed Synthesis of Aliphatic Amines. Chem. Rev. 2020, 120, 2613-2692.

(2) (a) Nugent, T. C.; El-Shazly, M. Chiral Amine Synthesis-Recent Developments and Trends for Enamide Reduction, Reductive Amination, and Imine Reduction. Adv. Synth. Catal. 2010, 352, 753-819; (b) Xie, J.-H.; Zhu, S.-F.; Zhou, Q.-L. Transition Metal-Catalyzed Enantioselective Hydrogenation of Enamines and Imines. Chem. Rev. 2011, 111, 1713-1760; (c) Seo, C. S. G.; Morris, R. H. Catalytic Homogeneous Asymmetric Hydrogenation: Successes and Opportunities. Organometallics 2019, 38, 47-65.

(3) Kobayashi, S.; Mori, Y.; Fossey, J. S.; Salter, M. M. Catalytic Enantioselective Formation of C-C Bonds by Addition to Imines and Hydrazones: A Ten-Year Update. Chem. Rev. 2011, 111, 2626-2704.

(4) (a) Miyabe, H.; Ushiro, C.; Ueda, M.; Yamakawa, K.; Naito, T. Asymmetric Synthesis of $\alpha$-Amino Acids Based on Carbon Radical Addition to Glyoxylic Oxime Ether. J. Org. Chem. 2000, 65, 176-185; (b) Friestad, G. K.; Shen, Y.; Ruggles, E. L. Enantioselective Radical Addition to N-Acyl Hydrazones Mediated by Chiral Lewis Acids. Angew. Chem. Int. Ed. 2003, 42, 5061-5063; (c) Cho, D. H.; Jang, D. O. Enantioselective radical addition reactions to the $\mathrm{C}-\mathrm{N}$ bond utilizing chiral quaternary ammonium salts of hypophosphorous acid in aqueous media. Chem. Commun. 2006, 5045-5047; (d) Lee, S.; Kim, S. Enantioselective radical addition reactions to imines using binaphthol-derived chiral N-triflyl phosphoramides. Tetrahedron Lett. 2009, 50, 3345-3348.

(5) (a) Liu, R. Y.; Buchwald, S. L. CuH-Catalyzed Olefin Functionalization: From Hydroamination to Carbonyl Addition. Acc. Chem. Res. 2020, 53, 1229-1243; (b) Yang, Y.; Shi, S.-L.; Niu, D.; Liu, P.; Buchwald, S. L. Catalytic asymmetric hydroamination of unactivated internal olefins to aliphatic amines. Science 2015, 349, 62-66; (c) Niu, D.; Buchwald, S. L. Design of Modified Amine Transfer Reagents Allows the Synthesis of $\alpha-$ Chiral Secondary Amines via CuH-Catalyzed Hydroamination. J. Am. Chem. Soc. 2015, 137, 9716-9721.

(6) (a) Zhu, S.; Niljianskul, N.; Buchwald, S. L. Enantio- and Regioselective CuH-Catalyzed Hydroamination of Alkenes. J. Am. Chem. Soc. 2013, 135, 15746-15749; (b) Zhu, S.; Buchwald, S. L. Enantioselective CuH-Catalyzed Anti-Markovnikov Hydroamination of 1,1-Disubstituted Alkenes. J. Am. Chem. Soc. 2014, 136, 15913-15916; (c) Shi, S.-L.; Buchwald, S. L. Copper-catalysed selective hydroamination reactions of alkynes. Nat. Chem. 2015, 7, 38-44.

(7) (a) Lu, X.; Xiao, B.; Zhang, Z.; Gong, T.; Su, W.; Yi, J.; Fu, Y.; Liu, L. Practical carbon-carbon bond formation from olefins through nickel-catalyzed reductive olefin hydrocarbonation. Nat. Commun. 2016, 7, 11129; (b) Lu, X.; Xiao, B.; Liu, L.; Fu, Y. Formation of C( $\left.\mathrm{sp}^{3}\right)-\mathrm{C}\left(\mathrm{sp}^{3}\right)$ Bonds through Nickel-Catalyzed Decarboxylative Olefin Hydroalkylation Reactions. Chem. Eur. J. 2016, 22, 11161-11164; (c) Sun, S.-Z.; Börjesson, M.; MartinMontero, R.; Martin, R. Site-Selective Ni-Catalyzed Reductive Coupling of $\alpha$-Haloboranes with Unactivated Olefins. J. Am. Chem. Soc. 2018, 140, 12765-12769; (d) Zhou, F.; Zhu, J.; Zhang, Y.; Zhu, S. NiH-Catalyzed Reductive Relay Hydroalkylation: A Strategy for the Remote C(sp3)-H Alkylation of Alkenes. Angew. Chem. Int. Ed. 2018, 57, 4058-4062; (e) Bera, S.; Hu, X. Nickel-Catalyzed Regioselective Hydroalkylation and Hydroarylation of Alkenyl Boronic Esters. Angew. Chem. Int. Ed. 2019; (f) Qian, D.; Hu, X. Ligand-Controlled Regiodivergent Hydroalkylation of Pyrrolines. Angew. Chem. Int. Ed. 2019, 58, 18519-18523; (g) Sun, S.-Z.; Romano, C.; Martin, R. Site-Selective Catalytic Deaminative Alkylation of Unactivated Olefins. J. Am. Chem. Soc. 2019, 141, 16197-16201.

(8) (a) $\mathrm{Hu}, \mathrm{X}$. Nickel-catalyzed cross coupling of non-activated alkyl halides: a mechanistic perspective. Chem. Sci. 2011, 2, 1867-1886; (b) Choi, J.; Fu, G. C. Transition metal-catalyzed alkyl-alkyl bond formation: 
another dimension in cross-coupling chemistry. Science 2017, 356, eaaf7230; (c) Fu, G. C. Transition-Metal Catalysis of Nucleophilic Substitution Reactions: A Radical Alternative to $\mathrm{S}_{\mathrm{N}} 1$ and $\mathrm{S}_{\mathrm{N}} 2$ Processes. ACS Cent. Sci. 2017, 3, 692-700.

(9) (a) Wang, Z.; Yin, H.; Fu, G. C. Catalytic enantioconvergent coupling of secondary and tertiary electrophiles with olefins. Nature 2018, 563, 379-383; (b) Zhou, F.; Zhang, Y.; Xu, X.; Zhu, S. NiH-Catalyzed Remote Asymmetric Hydroalkylation of Alkenes with Racemic $\alpha$-Bromo Amides. Angew. Chem. Int. Ed. 2019, 58, 1754-1758; (c) He, S.-J.; Wang, J.-W.; Li, Y.; Xu, Z.-Y.; Wang, X.-X.; Lu, X.; Fu, Y. Nickel-Catalyzed Enantioconvergent Reductive Hydroalkylation of Olefins with $\alpha$-Heteroatom Phosphorus or Sulfur Alkyl Electrophiles. J. Am. Chem. Soc. 2020, 142, 214-221; (d) Yang, Z.-P.; Fu, G. C. Convergent Catalytic Asymmetric Synthesis of Esters of Chiral Dialkyl Carbinols. J. Am. Chem. Soc. 2020, 142, 5870-5875.

(10) (a) Vasseur, A.; Bruffaerts, J.; Marek, I. Remote functionalization through alkene isomerization. Nat. Chem.2016, 8, 209-219; (b) Sommer, H.; Juliá-Hernández, F.; Martin, R.; Marek, I. Walking Metals for Remote Functionalization. ACS Cent. Sci. 2018, 4, 153-165.

(11) Bera, S.; Mao, R.; Hu, X: Enantioselective $\mathrm{C}\left(\mathrm{sp}^{3}\right)-\mathrm{C}\left(\mathrm{sp}^{3}\right)$ Cross-Coupling of Non-activated Alkyl Electrophiles via Nickel Hydride Catalysis. ChemRxiv. Preprint. 2020, https://doi.org/10.26434/chemrxiv.12040398.v1.

(12) He, Y.; Liu, C.; Yu, L.; Zhu, S. Enantio- and Regioselective NiH-Catalyzed Reductive Hydroarylation of Vinylarenes with Aryl Iodides. Angew. Chem. Int. Ed. 2020, doi:.10.1002/anie.202010386.

(13) (a) Mecozzi, T.; Petrini, M. A Stereoselective Synthesis of Primary (Z)-Enecarbamates from $\alpha$ Amidoalkylphenyl Sulfones. Synlett 2000, 73-74; $\quad$ (b) Gooßen, L. J.; Salih, K. S. M.; Blanchot, M. Synthesis of Secondary Enamides by Ruthenium-Catalyzed Selective Addition of Amides to Terminal Alkynes. Angew. Chem. Int. Ed. 2008, 47, 8492-8495; (c) Trost, B. M.; Cregg, J. J.; Quach, N. Isomerization of N-Allyl Amides To Form Geometrically Defined Di-, Tri-, and Tetrasubstituted Enamides. J. Am. Chem. Soc. 2017, 139, 5133-5139.

(14) Cuesta-Galisteo, S.; Schörgenhumer, J.; Wei, X.; Merino, E.; Nevado, C. Nickel-Catalyzed Asymmetric Synthesis of $\alpha$-Arylbenzamides. Angew. Chem. Int. Ed. 2020, doi:. 10.1002/anie.202011342.

(15) (a) Cordier, C. J.; Lundgren, R. J.; Fu, G. C. Enantioconvergent Cross-Couplings of Racemic Alkylmetal Reagents with Unactivated Secondary Alkyl Electrophiles: Catalytic Asymmetric Negishi $\alpha-$ Alkylations of N-Boc-pyrrolidine. J. Am. Chem. Soc. 2013, 135, 10946-10949; (b) Mu, X.; Shibata, Y.; Makida, Y.; Fu, G. C. Control of Vicinal Stereocenters through Nickel-Catalyzed Alkyl-Alkyl Cross-Coupling. Angew. Chem. Int. Ed. 2017, 56, 5821-5824; (c) Huo, H.; Gorsline, B. J.; Fu, G. C. Catalyst-controlled doubly enantioconvergent coupling of racemic alkyl nucleophiles and electrophiles. Science 2020, 367, 559-564.

(16) (a) Boyington, A. J.; Seath, C. P.; Zearfoss, A. M.; Xu, Z.; Jui, N. T. Catalytic Strategy for Regioselective Arylethylamine Synthesis. J. Am. Chem. Soc. 2019, 141, 4147-4153; (b) Wei, X.; Shu, W.; GarcíaDomínguez, A.; Merino, E.; Nevado, C. Asymmetric Ni-Catalyzed Radical Relayed Reductive Coupling. J. Am. Chem. Soc. 2020, 142, 13515-13522.

(17) (a) Bethell, P. H.; Goad, L. J.; Evershed, R. P.; Ottaway, J. The Study of Molecular Markers of Human Activity: The Use of Coprostanol in the Soil as an Indicator of Human Faecal Material. J. Archaeol. Sci. 1994, 21,619-632; (b) Mander; L. N. Comprehensive natural products II: Chemistry and Biology; Elsevier: Amsterdam, 2010; (c) Mitchell, J. A.; Akarasereenont, P.; Thiemermann, C.; Flower, R. J.; Vane, J. R. Selectivity of nonsteroidal antiinflammatory drugs as inhibitors of constitutive and inducible cyclooxygenase. Proc. Natl. Acad. Sci. U. S. A. 1993, 90, 11693-11697.

(18) (a) Etxebarria, J.; Vicario, J. L.; Badía, D.; Carrillo, L. A general and enantiodivergent method for the asymmetric synthesis of piperidine alkaloids: concise synthesis of (R)-pipecoline, (S)-coniine and other 2alkylpiperidines. Tetrahedron 2007, 63, 11421-11428; (b) Bahamonde, A.; Al Rifaie, B.; Martín-Heras, V.; Allen, J. R.; Sigman, M. S. Enantioselective Markovnikov Addition of Carbamates to Allylic Alcohols for the Construction of $\alpha$-Secondary and $\alpha$-Tertiary Amines. J. Am. Chem. Soc. 2019, 141, 8708-8711; (c) Zhu, Q.; Lu, Y. Enantioselective Conjugate Addition of Nitroalkanes to Vinyl Sulfone: An Organocatalytic Access to Chiral Amines. Org. Lett. 2009, 11, 1721-1724. 